\title{
Circulating ErbB3/HER3 Levels are Associated with Increased Risk of Hypertension with Overweight: A Cross-Sectional Study
}

\section{Lijun Zhu}

Wannan Medical College https://orcid.org/0000-0002-2147-9407

\section{Zhengmei Fang}

Wannan Medical College

\author{
Yuelong Jin \\ Wannan Medical College \\ Weiwei Chang \\ Wannan Medical College \\ Mengyun Huang \\ Wannan Medical College

\section{Yan Chen} \\ Wannan Medical College \\ Yingshui Yao ( $\nabla$ yingshuiyao@126.com ) \\ Wannan Medical College
}

\section{Research}

Keywords: ErbB3, Hypertension, Overweight, Body mass index

Posted Date: September 3rd, 2020

DOI: https://doi.org/10.21203/rs.3.rs-70059/v1

License: (c) (1) This work is licensed under a Creative Commons Attribution 4.0 International License.

Read Full License 
1 Circulating ErbB3/HER3 levels are associated with increased risk of

2 hypertension with overweight: a cross-sectional study

3

4 Lijun Zhu ${ }^{1,2}$, Zhengmei Fang ${ }^{1,2}$, Yuelong Jin $^{1,2}$, Weiwei Chang ${ }^{1,2}$, Mengyun Huang ${ }^{1}$,

$5 \quad$ Yan Chen $^{1,2 *}$, Yingshui Yao ${ }^{1,2,3^{*}}$

61 Department of Epidemiology and Biostatistics, School of Public Health, Wannan

$7 \quad$ Medical College, Wuhu 241002, China

82 Institute of Chronic Disease Prevention and Control, Wannan Medical

9 College, Wuhu241002, China

103 Department of Medicine, AnHui College of Traditional Chinese Medicine, Wuhu 11241003 , China.

Lijun Zhu and Zhengmei Fang contributed equally to the work.

Address for correspondence:

Yingshui Yao, Department of Epidemiology and Biostatistics, School of Public Health, and Institute of Chronic Disease Prevention and Control, Wannan Medical College, No. 22, Wenchang Road, Wuhu 241002, Anhui, China. Department of Medicine, AnHui College of Traditional Chinese Medicine, No.18, Wuxia Shanxi Road, Wuhu 241002, Anhui, China. Phone: +86 553 3932466, E-mail:yingshuiyao@163.com Yan Chen, Department of Epidemiology and Biostatistics, School of Public Health, and Institute of Chronic Disease Prevention and Control, Wannan Medical College, 
No. 22, Wenchang Road, Wuhu 241002, Anhui, China. Phone: +86 553 3932466, E-mail: bingyan-1209@163.com

\section{Abstract}

Background: Hypertension and overweight are both independent risk factors for cardiovascular disease, and being overweight can more likely to develop high blood pressure. Recent research has shown that ErbB3/HER3 played a considerable role in the development of cardiovascular disease. However, ErbB3 levels effects in the hypertensive overweight patients are unknown. This study aimed to assess the association between ErbB3 levels and hypertension with overweight in Chinese population.

Methods: 128 Chinese adults aged 33-79 years, both sexes, underwent evaluation of height and weight, blood pressure, biochemical indicators and ErbB3 levels. Plasma ErbB3 levels was assessed by the Enzyme-linked immunosorbent assay (ELISA), and body mass index (BMI) was calculated as body weight divided by height squared. Participants were allocated in three groups according to blood pressure and BMI: healthy control group (CNT; $\mathrm{n}=31$; normotensive and nonoverweight), hypertension group (HT; $\mathrm{n}=33$; hypertension and nonoverweight) and hypertension with overweight group (HTO; $n=64$; hypertension and overweight). A 2-tailed $\mathrm{P}<0.05$ was defined to be statistically significant.

Results: The difference in mean of ErbB3 levels in three groups was not significant, but had a linear decrease in males, in CNT (1.13 \pm 0.36$)$, HT (1.03 \pm 0.36$)$ and HTO 
$(0.84 \pm 0.26) \mathrm{ng} / \mathrm{ml}$, with $\mathrm{P}$ was 0.007. In drinking population, the ErbB3 was significantly reduced in the HTO group as compared with the CNT and HT groups $(0.76 \pm 0.23$ versus $1.18 \pm 0.37$ and $1.20 \pm 0.30$, respectively). ErbB3 levels was negatively correlated with DBP in males $(\mathrm{r}=-0.293, \mathrm{P}=0.012)$, in smoking populations $(\mathrm{r}=-0.47, \mathrm{P}=0.004)$ and drinking populations $(\mathrm{r}=-0.387, \mathrm{P}=0.008)$. Besides, BMI in males and in drinking populations and UA in males presented negatively correlations with ErbB3 $(\mathrm{p}<0.05)$. The multivariate conditional logistic regression showed that plasma ErbB3 levels was associated with reduced risk for HTO in males (OR 0.054; 95\% CI: 0.007-0.412) and drink group(OR 0.002; 95\% CI: 0.000-0.101).

Conclusions: The apparent association between lower ErbB3 levels and overweight hypertensive patients suggested that ErbB3 may contribute to the pathogenesis to hypertension with overweight, with BMI, gender, and drinking all potentially modulating the process.

Keywords: ErbB3; Hypertension; Overweight; Body mass index.

\section{Background}

Hypertension is a condition in which the blood vessels have persistently raised pressure, and it is one of the most pressing public health challenges. The global burden of hypertension has been growing over time, largely driven by population growth, changes in lifestyle, and aging [1]. From 1975 to 2015, the number of adults with raised blood pressure increased from 594 million to 1.13 billion, with the increase largely in low-income and middle-income countries[2]. On the basis of data 
from 451755 Chinese, the prevalence of hypertension among adults was $27.9 \%$ and was similar among men (28.6\%) and women (27.2\%) [3]. Previous studies showed that the prevalence of hypertension increased perhaps could be accounted for by increasing body mass index (BMI)[4]. Raised BMI is a major risk factor for a number of chronic diseases, including diabetes, cardiovascular diseases and hypertension [5]. Several epidemiological studies have shown an association between BMI and blood pressure in normal weight and overweight patients [6]. Previous studies have reported that BMI is strongly associated with hypertension in northern Chinese adults [7] and BMI can explain $45 \%$ of the age-adjusted increase in DBP over the period in Indians [8].

The ErbB/HER family of membrane-bound tyrosine kinase receptors comprises ErbB1, ErbB2, ErbB3, and ErbB4, which activate potent signaling pathways that mediate cell proliferation or differentiation[9]. Unlike other ErbB family members, ErbB3/HER3 has been investigated less frequently because its intracellular kinase domain is thought to be an inactive pseudokinase, where its function depends on interactions with ErbB partners. Therefore, functional in ErbB3 have been neglected for this pseudo-kinase [10]. Currently, although ErbB3 is widely used as a tumor marker, it has also been reported to associated with cardiovascular disease recently[11].

Monocyte surface ErbB3 mRNA expression was inversely correlated in subjects with heart failure but not in human subjects without heart failure[12]. In addition, the brown fat-enriched secreted factor $\mathrm{Nrg} 4$ has the potential for the treatment of 
obesity-associated disorders and primarily signals through ErbB3 to regulate diverse biological processes [13]. Emerging evidence suggested that dysregulation of the ErbB3 appears important in mediating hyperglycemia-induced vascular dysfunction [14]. A case-control study has showed that ErbB3 genetic polymorphisms was associated with pathogenesis of coronary artery disease[15]. Recent animal study has found that transient receptor potential vanilloid 4 (TRPV4) ion channels, a major $\mathrm{Ca} 2+$ influx pathway in endothelial cells, were impaired contributes to obesity-induced hypertension [16]. Moreover, G Protein-Coupled Receptors can regulator TRPV4 action in the vasculature by mediating ErbB family transactivation [17]. Thus, we assumed that the ErbB3 may participate in the process that leads to the occurrence and development of hypertension and overweight.

In view of ErbB3 may play a considerable role in the development of cardiovascular disease. The aim of this study was to assess the relationship between plasma ErbB3 levels and hypertension with overweight in a Chinese adult population, and to provide the basis for the pathogenesis of hypertension with overweight.

\section{Methods}

\section{Study Subjects}

The subjects were recruited via Health Management Center \& Physical Examination Center at Yijishan Hospital of Wannan Medical College from July 2019 to August 2019. A total of 128 adults aged 33-79 years were investigated. According to "Seventh report of the joint national committee on prevention, detection, evaluation, and 
111 treatment of high blood pressure", Hypertension is generally defined as a systolic 112 blood pressure of $\geq 140 \mathrm{~mm} \mathrm{Hg}$ or a diastolic blood pressure of $\geq 90 \mathrm{~mm} \mathrm{Hg}$ or use of 113 antihypertensive medications. For adults, WHO defines overweight as follows:

114 overweight is a BMI greater than or equal to 25 . In this study, healthy control group 115 (CNT) was defined as both normotensive and BMI < 25; hypertension group (HT) 116 was defined as both hypertensive and BMI $<25$, and hypertension with overweight 117 group (HTO) was defined as both hypertensive and BMI $\geq 25$. Finally, 128 adults, 118 including 31 controls, 33 hypertensives, and 64 hypertension with overweight subjects 119 were selected to assess the association between plasma ErbB3 levels and hypertension with overweight. consent was obtained from all participants.

\section{Data collection}

At physical examinations, all subjects were measured for height and weight, and blood pressure. Body mass index (BMI) was calculated as body weight (kg) divided by height squared $\left(\mathrm{m}^{2}\right)$. A well-trained research staff measured blood pressure once using electronic sphygmomanometer with the participant in the sitting position after at current and ex-drinkers) or non-drinkers. 
Blood samples were obtained from the subjects fasted overnight for at least $10 \mathrm{~h}$. Total cholesterol (TC), triglyceride (TG), high-density lipoprotein cholesterol (HDL-C), low -density lipoprotein cholesterol (LDL-C) levels, glucose (GLU), and uric acid (UA) were all measured using standard methods by the physical examination institution.

\section{Enzyme-linked immunosorbent assay (ELISA)}

A 3-ml venous blood samples was collected and centrifugated at 3000r/min for 10 min. The supernatant was collected using a micropipette and stored in the refrigerator at $-80^{\circ} \mathrm{C}$ for use. The plasma samples were then thawed at room temperature for ELISA which performed using a commercial kit (Catalogue No.CSB-EL007765HU).The method is operated according to the manufacturers' instructions, and was repeated twice to calculate the average of two parallel samples.

\section{Statistical analysis}

Statistical analysis was carried out with the SPSS software (version 18.0, SPSS Inc., Chicago, IL). The normality assumption of the data was determined using the Kolmogorov Smirnov test. Quantitative data were expressed as mean \pm standard deviation (mean $\pm \mathrm{SD}$ ), and categorical data are expressed as frequency (n and \%). The differences for variables among the groups were determined by one-way analysis of variance (ANOVA) or Chi-square test. Pearson's correlation was used for parametric correlations. Multiple unconditional logistic regression analysis with effect ratios [odds ratio (OR) and 95\% confidence intervals (CI)] was applied to estimate the risk of hypertension, hypertension with overweight after adjusting for potential 
confounding factors. A 2-tailed $\mathrm{P}<0.05$ was defined to be statistically significant.

\section{Results}

\section{Characteristics of the study participants}

As shown in Table 1, there was no differences in age, TC, LDL-C, HDL-C, Glu and the distribution of gender, drinking status among the three groups $(\mathrm{P}>0.05)$. The TG significantly increased in the HT group in comparison with the CNT group, while was decreased comparison with the HTO group. Compared with the HT group, the UA levels in the HTO group was increased.

\section{Comparison of plasma levels of ErbB3 among the three groups}

There were no statistical differences in ErbB3 levels between the three groups. By gender subgroup, the plasma concentrations of ErbB3 $(1.13 \pm 0.36,1.03 \pm 0.36$, $0.84 \pm 0.26) \mathrm{ng} / \mathrm{ml}$ had a linear decrease in CNT group, HT group and HTO group in males, with $\mathrm{P}$ was 0.007. In subgroup of drinking population, the ErbB3 was significantly decreased in the HTO group as compared with the CNT and HT groups $(0.76 \pm 0.23$ versus $1.18 \pm 0.37$ and $1.20 \pm 0.30$, respectively) (Table2).

\section{Correlation of plasma ErbB3 levels and clinical characteristics}

Table 3 summarizes Pearson's correlation coefficients between ErbB3 and hypertension risk factors, stratified by gender, smoking and drinking. Correlation analysis showed that the ErbB3 levels was negatively correlated with DBP in males( $\mathrm{r}=-0.293, \mathrm{P}=0.012)$, in smoking populations $(\mathrm{r}=-0.47, \mathrm{P}=0.004)$ and drinking populations $(\mathrm{r}=-0.387, \mathrm{P}=0.008)$. Besides, it is noteworthy that $\mathrm{BMI}$ both in males and 
176 in drinking population and UA in males presented negatively correlations with ErbB3

$177 \quad($ all $\mathrm{P}<0.05)$.

178 Multinomial logistic regression

179 In Table 4, we examined the OR of HT and HTO associated with increasing levels of

180 ErbB3 within subgroups of gender, current smoking and drinking. Plasma ErbB3

181 levels was negatively associated with hypertension with overweight, with OR (95\%

$182 \mathrm{CI})$ was $0.054(0.007-0.412)$, and 0.002(0.000-0.101), for males and drink group,

183 respectively. After adjustment for age, gender, the association remains significant.

\section{Discussion}

Framingham Study have shown that hypertension[18] and overweight[19] are both independent risk factors for cardiovascular disease, and about $30 \%$ of hypertensive individuals can be classified as being obese[20]. In addition, ErbB3 has been reported to play an important role in the maintenance and development of cardiovascular disorders $[11,15]$. This study is the first to demonstrate the importance of ErbB3 levels in hypertension with overweight. We observed the ErbB3 levels had negatively associations with hypertension with overweight in males and drinking populations. These results indicated that the ErbB3 levels might contribute to the development of blood pressure regulation through NRG-1/ErbB signaling as an antihypertensive system, but this effect is probably not that strong[21]. We observed a negative 
association between the ErbB3 and DBP in the males, drinking and smoking groups in this study. Previous reports suggested that males were generally at greater risk for hypertension [22] than were age-matched females. Another study reported that environmental exposures to drinking, smoking were associated with adult hypertension in Japanese population [23]. Remarkably, DBP has increased sensitivity to environmental changes, and more strongly predicts cardiovascular disease risk in younger Chinese adulthood[24]. In the present study, BMI both in males and in drinking population and UA in males presented negatively correlations with ErbB3. Males were more overweight, and consumed more alcohol than females [25]. Additionally, Genetic studies have shown ErbB3 gene is responsible for variation in the LDL-C serum concentration, is known to be involved in lipid homeostasis and obesity [26]. Taken together, BMI, gender, and drinking all potentially modulate the association that ErbB3 Levels and hypertension with overweight.

However, the mechanisms that link ErbB3 and hypertension and overweight are currently unclear. Previous studies have explored potential mechanisms linking adiposity and high BP, including sympathetic nervous system activation, activation of the renin-angiotensin system, inflammatory responses and insulin resistance[27]. It may be speculated that ErbB3 participating in networks related to lipid metabolism which is known a major variable in the etiology of overweight and hypertension[28]. Beside, ErbB3 participate in neutrophil survival and ErbB3 inhibitors play positive roles in accelerating inflammation resolution[29], which is known predisposing factor for development and progression of hypertension and overweight. Signaling 
interactions have been reported between ErbB3 family members and IGF-IR[30], and

221 our group has previously shown that IGF-IR may contribute to the genetic

222 susceptibility to hypertension[31], and the higher IGF-IR mRNA expression observed

223 in obese children[32]. It is reasonable to believe that the ErbB3 levels play an

224 important role in the regulation of hypertension with overweight by effecting on

225 IGF-IR.

226 There are a few limitations to our study. Firstly, the potential bias in

227 cross-sectional study often distorted results for epidemiological association studies.

228 Secondly, no significant differences in the plasma ErbB3 levels were observed among

229 the three groups in the total population, despite the fact that strict standards were used

230 to select representative cases and controls. Relatively small sample size may have led

231 to weak statistical power. Thus, further prospective studies are required to confirm the

232 observations, and increase the sample size and research involving obese population

233 studies are warranted

234 Conclusions

235 In summary, our results demonstrated for the first time that expression of ErbB3

236 levels was significantly down-regulated in hypertension with overweight, and BMI,

237 gender, and drinking all potentially modulating the process. Therefore, it is of great

238 value to prevent the development of hypertension with overweight by guiding the

239 early and correct management of ErbB3 levels.

\section{Abbreviations}

241 CNT: Healthy control group; HT: Hypertension group; HTO: Hypertension with 
overweight group; BMI : Body mass index; SBP: Systolic blood pressure; DBP: Diastolic blood pressure; ELISA: Enzyme-linked immunosorbent assay; GLU: Glucose; TC: Total cholesterol; TG: Triglycerides; HDL-C: High-density lipoprotein cholesterol; LDL-C: Low-density lipoprotein cholesterol; UA: Uric acid.

\section{Ethics approval and consent to participate}

The study was approved by the Ethics Committee of the First Affiliated Yijishan Hospital of Wannan Medical College (Wuhu,China).

\section{Consent for publication}

All authors agree the publication of this paper.

\section{Availability of data and materials}

The datasets during and/or analyzed during the current study will be available from the corresponding author on reasonable request.

\section{Competing interests}

The authors declare no conflict of interest.

\section{Funding}

This research was supported by the National Natural Science Foundation of China (No. 81874280 and No. 81673266); The Fifth Batch of Talents Selected under the Special Support Plan in Anhui Provence(Organization Department of Anhui provincial Party committee,[2019]No.14); Anhui Provincial Natural Science Foundation (No. 1808085QH283 and No. 1808085MH297); Key Projects of Anhui Provincial Department of Education (No. KJ2019A0404 and No. KJ2019A0405)

\section{Authors' contributions}


Lijun Zhu and Zhengmei Fang conducted the experiments. Lijun Zhu managed and analyzed the data and wrote the manuscript. Mengyun Huang was one of the principal investigators of the study. Yuelong Jin revised the manuscript. Weiwei Chang was a contributor in organizing the database. Yan Chen and Yingshui Yao reviewed the manuscript. All authors read and approved the final manuscript.

\section{Acknowledgments}

\section{Not applicable}

\section{Authors' information}

${ }^{1}$ Department of Epidemiology and Biostatistics, School of Public Health, Wannan Medical College, Wuhu 241002, China. ${ }^{2}$ Institute of Chronic Disease Prevention and Control, Wannan Medical College ,Wuhu241002, China. ${ }^{3}$ Department of Medicine, AnHui College of Traditional Chinese Medicine, Wuhu 241003, China.

\section{Reference}

1. Lewington S, Lacey B, Clarke R, Guo Y, Kong XL, Yang L, Chen Y, Bian Z, Chen J, Meng J, et al: The Burden of Hypertension and Associated Risk for Cardiovascular Mortality in China. JAMA Intern Med 2016, 176:524-532.

2. Collaboration NCDRF: Worldwide trends in blood pressure from 1975 to 2015: a pooled analysis of 1479 population-based measurement studies with 19.1 million participants. Lancet 2017, 389:37-55.

3. Hu.ST, Gao.RL, Liu.LS, Zhu.ML, Wang.W, Wang.YJ, Wu.ZS, Li.HJ, Gu.DF, Yang.YJ, et al: Summary of report on cardiovascular diseases in China (2018). Chin Circ J 2019, 34:209-220.

4. Cutler JA, Sorlie PD, Wolz M, Thom T, Fields LE, Roccella EJ: Trends in hypertension prevalence, awareness, treatment, and control rates in United States adults between 1988-1994 and 1999-2004. Hypertension 2008, 52:818-827.

5. Wilson PW, D'Agostino RB, Sullivan L, Parise H, Kannel WB: Overweight and obesity as determinants of cardiovascular risk: the Framingham experience. Arch Intern Med 2002, 162:1867-1872.

6. Ma J, Wang Z, Dong B, Song Y, Hu P, Zhang B: Quantifying the relationships of blood 
pressure with weight, height and body mass index in Chinese children and adolescents. $J$ Paediatr Child Health 2012, 48:413-418.

7. Feng RN, Zhao C, Wang C, Niu YC, Li K, Guo FC, Li ST, Sun CH, Li Y: BMI is strongly associated with hypertension, and waist circumference is strongly associated with type 2 diabetes and dyslipidemia, in northern Chinese adults. J Epidemiol 2012, 22:317-323.

8. Linhart C, Tukana I, Lin S, Taylor R, Morrell S, Vatucawaqa P, Magliano D, Zimmet P: Continued increases in hypertension over three decades in Fiji, and the influence of obesity. J Hypertens 2016, 34:402-409; discussion 409.

9. Yarden Y, Sliwkowski MX: Untangling the ErbB signalling network. Nat Rev Mol Cell Biol 2001, 2:127-137.

10. Shi F, Telesco SE, Liu Y, Radhakrishnan R, Lemmon MA: ErbB3/HER3 intracellular domain is competent to bind ATP and catalyze autophosphorylation. Proc Natl Acad Sci U S A 2010, 107:7692-7697.

11. Hedhli N, Kalinowski A, K SR: Cardiovascular effects of neuregulin-1/ErbB signaling: role in vascular signaling and angiogenesis. Curr Pharm Des 2014, 20:4899-4905.

12. Ryzhov S, Matafonov A, Galindo CL, Zhang Q, Tran TL, Lenihan DJ, Lenneman CG, Feoktistov I, Sawyer DB: ERBB signaling attenuates proinflammatory activation of nonclassical monocytes. Am J Physiol Heart Circ Physiol 2017, 312:H907-H918.

13. Wang GX, Zhao XY, Meng ZX, Kern M, Dietrich A, Chen Z, Cozacov Z, Zhou D, Okunade $\mathrm{AL}, \mathrm{Su} X$, et al: The brown fat-enriched secreted factor $\mathrm{Nrg} 4$ preserves metabolic homeostasis through attenuation of hepatic lipogenesis. Nat Med 2014, 20:1436-1443.

14. Akhtar S, Chandrasekhar B, Attur S, Dhaunsi GS, Yousif MH, Benter IF: Transactivation of ErbB Family of Receptor Tyrosine Kinases Is Inhibited by Angiotensin-(1-7) via Its Mas Receptor. PLoS One 2015, 10:e0141657.

15. Maitusong B, Xie X, Ma YT, Fu ZY, Yang YN, Li XM, Liu F, Chen BD, Gai MT: Association between ErbB3 genetic polymorphisms and coronary artery disease in the Han and Uyghur populations of China. Int J Clin Exp Med 2015, 8:16520-16527.

16. Ottolini M, Hong K, Cope EL, Daneva Z, DeLalio LJ, Sokolowski JD, Marziano C, Nguyen NY, Altschmied J, Haendeler J, et al: Local Peroxynitrite Impairs Endothelial Transient Receptor Potential Vanilloid 4 Channels and Elevates Blood Pressure in Obesity. Circulation 2020, 141:1318-1333.

17. Saifeddine M, El-Daly M, Mihara K, Bunnett NW, McIntyre P, Altier C, Hollenberg MD, Ramachandran R: GPCR-mediated EGF receptor transactivation regulates TRPV4 action in the vasculature. Br J Pharmacol 2015, 172:2493-2506.

18. Kannel WB: Blood pressure as a cardiovascular risk factor: prevention and treatment. JAMA 1996, 275:1571-1576.

19. Hubert HB, Feinleib M, McNamara PM, Castelli WP: Obesity as an independent risk factor for cardiovascular disease: a 26-year follow-up of participants in the Framingham Heart Study. Circulation 1983, 67:968-977.

20. MacMahon S, Cutler J, Brittain E, Higgins M: Obesity and hypertension: epidemiological and clinical issues. Eur Heart J 1987, 8 Suppl B:57-70.

21. Matsukawa R, Hirooka Y, Nishihara M, Ito K, Sunagawa K: Neuregulin-1/ErbB signaling in rostral ventrolateral medulla is involved in blood pressure regulation as an antihypertensive system. J Hypertens 2011, 29:1735-1742. 
22. Wiinberg N, Hoegholm A, Christensen HR, Bang LE, Mikkelsen KL, Nielsen PE, Svendsen TL, Kampmann JP, Madsen NH, Bentzon MW: 24-h ambulatory blood pressure in 352 normal Danish subjects, related to age and gender. Am J Hypertens 1995, 8:978-986.

23. Shiue I: Self and environmental exposures to drinking, smoking, gambling or video game addiction are associated with adult hypertension, heart and cerebrovascular diseases, allergy, self-rated health and happiness: Japanese General Social Survey, 2010. Int J Cardiol 2015, 181:403-412.

24. Attard SM, Herring AH, Zhang B, Du S, Popkin BM, Gordon-Larsen P: Associations between age, cohort, and urbanization with SBP and DBP in China: a population-based study across 18 years. J Hypertens 2015, 33:948-956.

25. Freedman DS, Jacobsen SJ, Barboriak JJ, Sobocinski KA, Anderson AJ, Kissebah AH, Sasse EA, Gruchow HW: Body fat distribution and male/female differences in lipids and lipoproteins. Circulation 1990, 81:1498-1506.

26. Cox LA, Birnbaum S, VandeBerg JL: Identification of candidate genes regulating HDL cholesterol using a chromosomal region expression array. Genome Res 2002, 12:1693-1702.

27. Rahmouni K: Obesity-associated hypertension: recent progress in deciphering the pathogenesis. Hypertension 2014, 64:215-221.

28. Zou ZY, Yang YD, Wang S, Dong B, Li XH, Ma J: The importance of blood lipids in the association between BMI and blood pressure among Chinese overweight and obese children. Br J Nutr 2016, 116:45-51.

29. Rahman A, Henry KM, Herman KD, Thompson AA, Isles HM, Tulotta C, Sammut D, Rougeot JJ, Khoshaein N, Reese AE, et al: Inhibition of ErbB kinase signalling promotes resolution of neutrophilic inflammation. Elife 2019, 8.

30. Jin Q, Esteva FJ: Cross-talk between the ErbB/HER family and the type I insulin-like growth factor receptor signaling pathway in breast cancer. J Mammary Gland Biol Neoplasia 2008, 13:485-498.

31. Yao Y, Zhu L, Fang Z, Yang S, Chen Y, Jin Y, Zhao X, Zhao H, Zhuang Q, Chen X, Shen C: Insulin-Like Growth Factor-1 and Receptor Contribute Genetic Susceptibility to Hypertension in a Han Chinese Population. Am J Hypertens 2018, 31:422-430.

32. Ricco RC, Ricco RG, Queluz MC, de Paula MTS, Atique PV, Custodio RJ, Tourinho Filho H, Del Roio Liberatori R, Jr., Martinelli CE, Jr.: IGF-1R mRNA expression is increased in obese children. Growth Horm IGF Res 2018, 39:1-5. 